\title{
Ionosphere/Plasmasphere sounding with ground and space-based GNSS observations
}

\author{
Guillermo González-Casado, J. Miguel Juan, Jaume Sanz and Yixie Shao \\ Research Group of Astronomy and Geomatics, Technical University of Catalonia (gAGE/UPC) \\ Barcelona, Spain \\ guillermo.gonzalez@upc.edu
}

\begin{abstract}
Applying a methodology developed and tested in previous studies, the contribution from the ionospheric and plasmaspheric regions to the total electron content (measured by ground receivers) is analyzed. The method is based in the electron density profiles retrieved from radio occultations observed with low Earth orbit satellites, combined with an accurate empirical modeling of the topside-ionosphere electron density. The results of a climatological study of the fractional electron content from the ionospheric region are presented for a year of low solar activity. It is shown that a simple parametric model can be used to reproduce the electron content variations in the ionosphere and the plasmasphere between sunrise and midday, the period of the day showing the largest electron content variability.
\end{abstract}

Keywords-ionosphere; plasmasphere; radio occultations; total electron content

\section{INTRODUCTION}

Usually, in Global Navigation Satellite System (GNSS) applications, the ionosphere is modeled as a single layer located at a fixed altitude representative of that region [1]. However, this approach disregards the contribution to slant total electron content measurements from the plasmasphere, a region extending toward heights of several thousands of kilometers. Previous studies have shown that this contribution is not negligible, especially during the night [2]. Hence, the characterization of the separated electron content from the ionosphere and the plasmasphere is of great importance to achieve a precise modeling of the GNSS signal delays. Recently ([3], [4]), a method that self-consistently combines radio occultations (ROs) from the COSMIC/FORMOSAT-3 (CF3) constellation and global ionospheric maps (GIMs) [5] from the International GNNS Service (IGS) has been developed to achieve a reliable determination of the electron contents in the ionospheric and plasmaspheric regions separately.

In this study, we present preliminary results showing that the method can be used to derive some general trends characterizing the ionosphere-plasmasphere interplay, which can be reproduced by means of a simple parametric model.

\section{DATA SAMPLE AND METHOD}

The details of the methodology applied in this study can be found in [3] and [4], we only summarize here the details required to understand how the ionospheric and plasmaspheric electron contents are calculated and the data required to implement the method. First, the improved Abel transform inversion ([3], [6]) is used to retrieve electron density profiles from a data sample of RO measurements taken by the CF3 constellation. This improved retrieval technique takes into account the horizontal gradients in the electron density by using externally provided vertical total electron content (VTEC) values.

RO observations sampled altitudes from 200 to $700 \mathrm{~km}$ at least. The data sampled used in this study amounts to more than 70,000 ROs covering a period of one year, 2007, that was part of a particularly long and deep solar minimum, lasting over 4 years (from 2007 to 2010). On the other hand, VTEC values for the time intervals corresponding to different RO measurements were collected from the GIMs provided by IGS and subsequently interpolated to the different geographical locations covered by each RO in the sample. The data sample provides a reasonably dense coverage of all geographic locations around the globe.

After deriving the altitude variation of the electron density from every RO, the topside-electron density profile (altitude higher than $400 \mathrm{~km}$ typically) was modeled by means of the simplified topside ionosphere plus protonosphere (STIP) model [3]. The STIP model fit was used to extract the contribution from the bottom-side plasmaspheric electron density to the total electron density profile. After the bottom-side plasmaspheric electron density was separated, the remaining electron density profile was integrated to obtain the ionospheric electron content, $E C_{\text {ion }}$. Finally, after having calculated $E C_{\text {ion, }}$, the plasmaspheric electron content, $E C_{\mathrm{pl}}$, is calculated as the difference [4]:

$$
E C_{\mathrm{pl}}=V T E C-E C_{\mathrm{ion}}
$$

where $V T E C$ is the vertical total electron content at the longitude and latitude associated to the RO and having been previously used to retrieve the electron density profile.

\section{IONOSPHERE-PLASMASPHERE INTERPLAY}

The relationship between $E C_{\mathrm{pl}}$ and $E C_{\text {ion }}$ is of great interest to characterize the coupling and interplay of the ionosphereplasmasphere system. This relationship has been analyzed using the methodology described in the previous section. In 
particular, the fractional electron content in the ionosphere, $I O N_{f}=E C_{\text {ion }} / V T E C$, has been studied during 2007. It is evident that the magnitude of the $I O N_{f}$ variations will be significantly smaller than the $E C_{\text {ion }}$ variations, since the former is a fractional quantity. The variations of $I O N_{f}$ will be mostly driven by the large variations observed in the ionospheric electron content (see Figure 4), but typical trends of the fractional ionospheric electron content can be immediately associated to trends in the fractional plasmaspheric electron content, $P L_{f}=E C_{\mathrm{pl}} / V T E C$, through the relationship:

$$
I O N_{f}+P L_{f}=1
$$

Fig. 1 shows the frequency distribution of $I O N_{f}$ during summer and winter in 2007. One can see that the values of the fractional electron content in the ionosphere have a similar distribution in both seasons, with nearly the same median and peak values (around 0.7). In fact, about $75 \%$ of the measurements are within the range 0.6-0.8, suggesting that the midday variation of $I O N_{f}$ was not very large during 2007.

Fig. 2 shows a more detailed analysis of the local time (LT) variations of $I O N_{f}$ during summer and winter seasons for two different geomagnetic latitude intervals and in the two Earth hemispheres. One can see that the maximum mean value of $I O N_{f}$ (approximately equal to 0.7 ) is always achieved around midday, being similar in all the cases shown in the figure. The minimum value of $I O N_{f}$ occurs just before sunrise $\mathrm{LT}$ and is smaller around the geomagnetic equator than for mid-latitude. Sunrise LT in each graph of Figure 2 approximately coincides with the starting time of the $I O N_{f}$ enhancement from its minimum value. This enhancement clearly traces the recovering of the daytime ionosphere following the decrease of the solar zenith angle after sunrise. The ionosphere reaches a level of maximum ionization nearly at midday.

One can also observe in Fig. 2 that the slope of the LT variation of $I O N_{f}$ during a few hours after sunrise is similar in summer and winter seasons, but it is different for the middle and equatorial geomagnetic latitude ranges. In order to model this particular trend, the following function has been considered to fit the LT variation of $I O N_{f}$ between sunrise and midday:

$$
I O N_{f}(t)=I_{\max }-D \exp \left[-\left(t-t_{s}\right) / \tau_{i}\right]
$$

where $t$ indicates the LT, $t_{s}$ is the time of sunrise at $350 \mathrm{~km}$ of altitude (sunrise has been considered at a representative altitude of the ionospheric $\mathrm{F}$ layer), and $D=I_{\max }-I_{s}$ being the difference between the maximum and minimum values achieved by $I O N_{f}$, with $I_{s}=I O N_{f}\left(t_{s}\right)$.

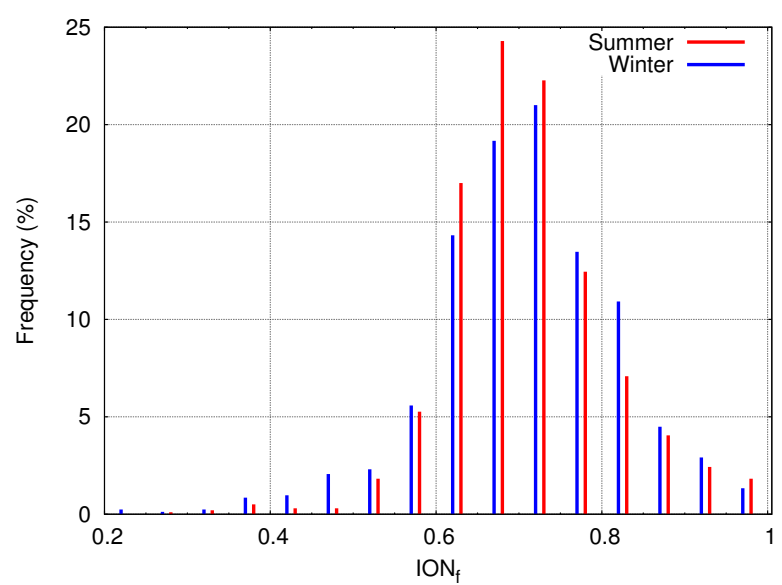

Fig. 1. Frequency distribution of the fractional electron content in the ionosphere at the North hemisphere (from 0 to 60 degrees of geomagnetic latitude) and around midday (LT interval from 11:00 to 15:00).
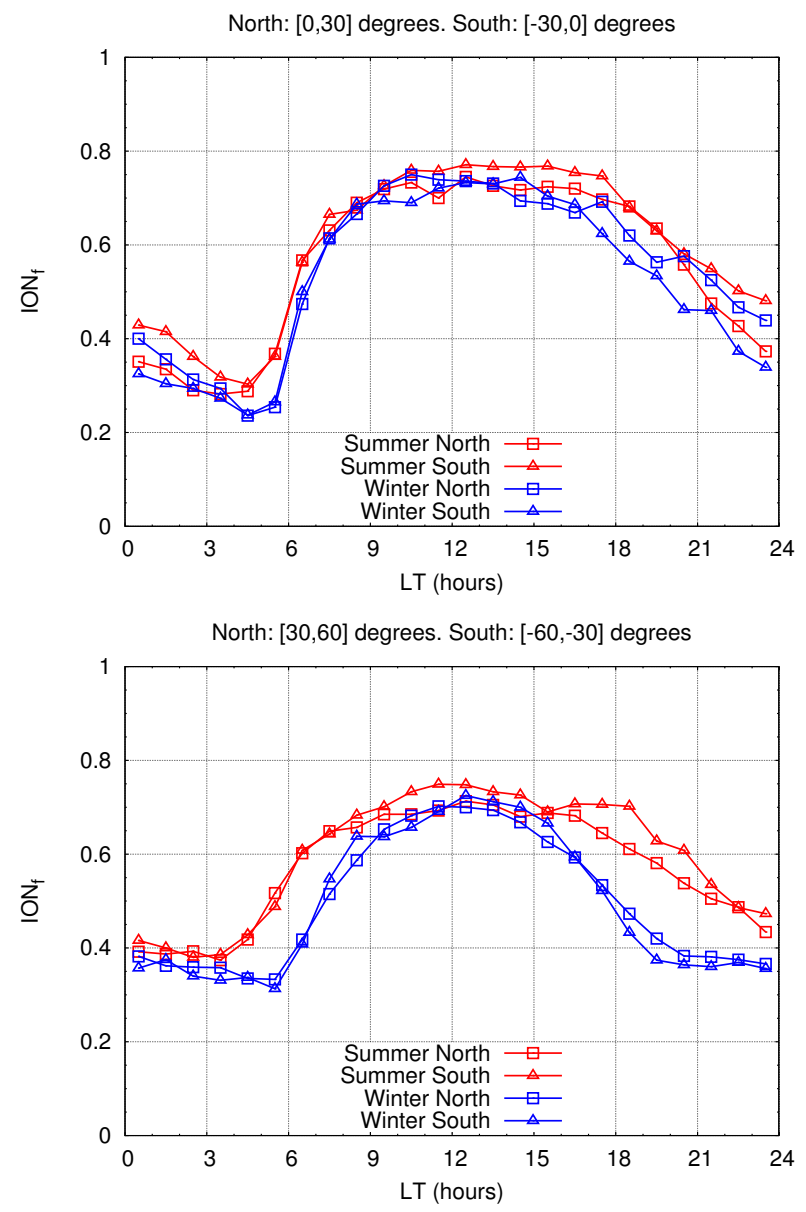

Fig. 2. LT variation of the hourly-mean value of $I O N_{f}$ during the summer and winter seasons in the Northern and Southern hemispheres.

We have set $I_{\max }=0.7$ (in accordance with the results shown in Figures 1 and 2), so equation (3) has two free parameters, 
namely, $D$ (or equivalently, $I_{s}$ ) and $\tau_{i}$, this last parameter corresponding to the timescale of development of the daytime ionosphere.

Fig. 3 displays some examples of the best-fit curves (black solid lines) to the $I O N_{f}$ values derived from our sample of ROs (red crosses). The data used for the fits cover the period of solar light from $t_{s}$ to approximately 13:00 LT. As can be seen, the model function is able to reproduce the typical LT variation in $I O N_{f}$ between sunrise and midday. Specifically, the median deviation (in absolute value) between data points and best-fit curves is approximately $10 \%$ in all the cases analyzed.

Tables I and II show the best-fit parameters for the different geomagnetic latitude bands considered, in different hemispheres and for local summer and winter periods. The geomagnetic latitude dependence of the best-fit parameters is evident in both Tables. In particular, the fractional VTEC in the ionosphere at sunrise, $I_{s}$, clearly increases with geomagnetic latitude (Table II), while the timescale, $\tau_{i}$, decreases as the latitude range considered approaches the geomagnetic equator (Table I), implying a faster development of the daytime F layer near the equatorial band than at intermediate latitudes. In general the summer/winter difference between the best-fit values in Tables I and II is small and almost negligible compared with the variations that can be observed among different geomagnetic-latitude ranges. These quantitative results confirm the trends previously observed in Fig. 2.

\section{DISCUSSION}

It is remarkable that the maximum mean value reached by $I O N_{f}$ during the 24-hour period (Figure 1) is approximately the same (about 0.7) and occurs around noon, for the two geomagnetic-latitude ranges considered and for summer and winter. This implies a nearly constant minimum value of $P L_{f}$ at the same period of the day and, consequently, an approximately constant ratio between the ionospheric and the plasmaspheric electron contents around noon along the year, regardless of the geomagnetic latitude considered. This indicates the existence of a significant coupling between the electron contents in the ionosphere and the plasmasphere during the low solar activity period analyzed. This coupling deserves a more detailed analysis for different solar activity periods to confirm if it corresponds to some general trend of the ionosphere-plasmasphere system.

The LT variations of $I O N_{f}$ calculated from our data sample show that the plasmasphere has a greater relevance than the ionosphere during the night. In particular, just before sunrise the plasmasphere makes the largest contribution to the VTEC. This contribution can be nearly $75 \%$ of the VTEC around the geomagnetic equator and during winter. The minimum value of $I O N_{f}$ is smaller for low geomagnetic latitudes than in the mid-latitude region (see Table II). There are several reasons that may explain this latitudinal difference of $I_{s}$. For instance, the electrodynamics of the equatorial ionosphere has important effects on $I O N_{f}$ and, particularly, $\mathbf{E} \times \mathbf{B}$ drifts at low latitudes
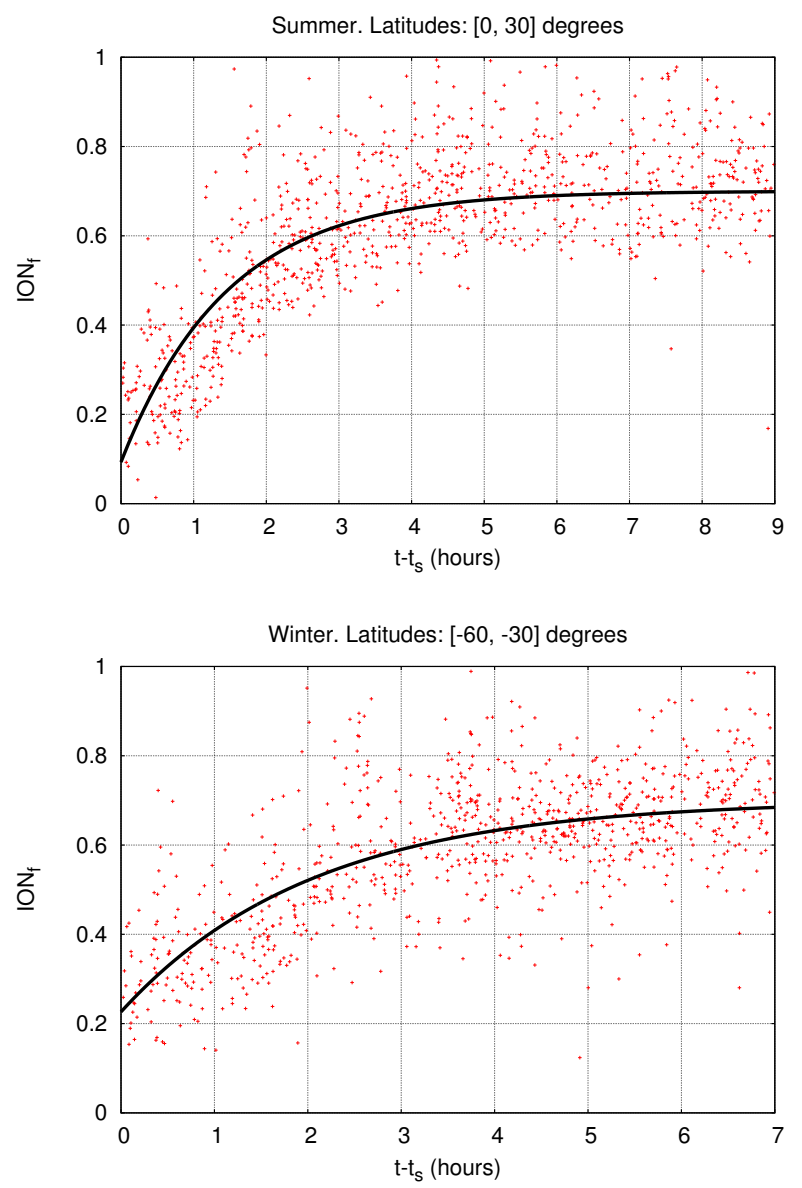

Fig. 3. Two examples of best-fit curves (black solid lines) to $I O N_{f}$ values (red crosses). The horizontal axis is the time after sunrise at $350 \mathrm{~km}$ of altitude.

during the nighttime are responsible of a downward ion drift that gives rise to a reduction of the equatorial density, while during the daytime the upward ion drift enhances the density in that region [7]. In this way, a larger contrast between the day and night $I O N_{f}$ values should be expected for low latitudes than for middle latitudes, as it is seen in Table II and in Fig. 2. Another effect that may contribute to reduce the value of $I_{s}$ from middle to low geomagnetic latitudes is the downward plasma flux during the night from the equatorial plasmasphere toward the mid-latitude ionosphere [2]. This plasma flux will enhance the pre-sunrise values of $I O N_{f}$ at intermediate geomagnetic latitudes.

Finally, the timescale $\tau_{i}$ of development of the daytime ionosphere has a marked latitudinal dependence (according to Fig. 2 and Table I), while for a given range of geomagnetic latitudes it is very similar between the two seasons of the year that have been analyzed. The faster development of the daytime equatorial ionosphere can be explained by the faster variation of the solar zenith angle after sunrise in this region. The time taken by the Sun to reach a given elevation is longer as the geographic latitude increases and, for this reason, during the first hours of the day the effects of the photoionization from 
the Sun will be noticeable near the equator faster than at intermediate and high latitudes. Consequently, one should expect that the timescale $\tau_{i}$ increases as the geographic latitude increases during summer and winter, but having similar values in both seasons. Although in Fig. 2 and Table I the data are grouped into intervals of geomagnetic (instead of geographic) latitude, this effect can still be observed.

\section{CONCLUSIONS}

A study of the electron content in the ionosphere and the plasmasphere based on ROs and VTEC measurements has been presented. The results have been derived using a novel technique that self-consistently combines an improved inversion technique for electron density retrieval from ROs, ground measurements of the VTEC and adequate modeling of the topside-ionosphere electron density. Applying this method to RO measurements from the $\mathrm{CF} 3$ satellite constellation and VTEC data from IGS GIMs during 2007, a global analysis of the plasmaspheric and ionospheric contributions to VTEC has been performed for a period of low solar and geomagnetic activity.

The results presented show that the contribution of the plasmasphere is, in general, significant and can even be the main contribution to the VTEC, particularly before dawn for low and intermediate geomagnetic latitudes. The ionosphere develops more abruptly (with a shorter timescale) and from a lower level of fractional electron content as the geomagnetic latitude decreases. However, the maximum value of $I O N_{f}$ (and the corresponding minimum value of $\left.P L_{f}\right)$ is typically the same for all ranges of geomagnetic latitudes considered and for summer and winter periods, the maximum being always achieved around noon, when the ionosphere reaches the state of maximum ionization. This implies that the ratio between the electron contents of the ionosphere and the plasmasphere around midday is quite constant, suggesting the existence of an important coupling between both regions.

A further analysis will be required to characterize in more detail the different dependencies of $I O N_{f}$ that have been observed, for example, considering different periods of solar and geomagnetic activity. The methodology applied in the present work certainly opens the possibility of conducting studies of the ionosphere-plasmasphere system at a global scale, which may ultimately lead to an improvement in the modeling of this coupled system. In particular, it has been shown that the model function given by equation (3) (with two free parameters) can be used to describe the typical time variation of the electron content during the development of the daytime ionosphere. For that period of time, equation (3) provides a simple tool to quickly calculate first-order approximations of the separate ionosphere and plasmasphere contributions from current VTEC maps. From this simple model one can develop more complex mapping functions taking into account the relevance of the plasmaspheric contribution to the VTEC and improving the reliability of current GIMs based on the assumption that the only contribution to VTEC measurements arises from a single layer representing the ionosphere.
TABLE I. BEST-FIT VALUES OF TIMESCALE IN HOURS

\begin{tabular}{|c|c|c|}
\hline $\begin{array}{c}\text { Geomagnetic } \\
\text { Latitude }\end{array}$ & Summer & Winter \\
\hline$\left[30^{\circ}, 60^{\circ}\right]$ & 2.2 & 2.4 \\
\hline$\left[0^{\circ}, 30^{\circ}\right]$ & 1.5 & 1.2 \\
\hline$\left[-30^{\circ}, 0^{\circ}\right]$ & 1.4 & 1.3 \\
\hline$\left[-60^{\circ},-30^{\circ}\right]$ & 1.9 & 2.1 \\
\hline
\end{tabular}

TABLE II. BEST-FIT VALUES OF MINIMUM IONOSPHERIC FRACTIONAL ELECTRON CONTENT

\begin{tabular}{|c|c|c|}
\hline $\begin{array}{c}\text { Geomagnetic } \\
\text { Latitude }\end{array}$ & Summer & Winter \\
\hline$\left[30^{\circ}, 60^{\circ}\right]$ & 0.26 & 0.29 \\
\hline$\left[0^{\circ}, 30^{\circ}\right]$ & 0.09 & 0.03 \\
\hline$\left[-30^{\circ}, 0^{\circ}\right]$ & 0.14 & 0.11 \\
\hline$\left[-60^{\circ},-30^{\circ}\right]$ & 0.24 & 0.23 \\
\hline
\end{tabular}

\section{ACKNOWLEDGMENTS}

The authors would like to thank the University Corporation for Atmospheric Research, the National Space Organization in Taiwan and CDAAC for making available the CF3 constellation data. We also thank IGS for the availability of their IONEX-format files from which VTEC values have been calculated.

\section{REFERENCES}

[1] J. Sanz, J.M. Juan, and M. Hernández-Pajares, "GNSS Data Processing. Vol. 1: Fundamentals and Algorithms," ESA communications, ESTEC TM-23/1, Noordwijk, the Netherlands, 2013.

[2] H.-B. Lee, G. Jee, Y. H. Kim, and J. S. Shim, "Characteristics of global plasmaspheric TEC in comparison with the ionosphere simultaneously observed by Jason-1 satellite", Journal of Geophysical Research. Space Physics, vol. 118, pp. 935-946, doi: 10.1002/jgra.50130, 2013.

[3] G. González-Casado, J.M. Juan, J. Sanz, A. Rovira-Garcia and A. Aragon-Angel, "Ionospheric and plasmaspheric electron contents inferred from radio occultations and global ionospheric maps", Journal of Geophysical Research. Space Physics, vol. 120, pp. 5983-5997, doi: 10.1002/2014JA020807, 2015.

[4] G. González-Casado, J.M. Juan, M. Hernández-Pajares and J. Sanz, "Two-component model of topside-ionosphere electron density profiles retrieved from Global Navigation Satellite Systems radio occultations", Journal of Geophysical Research. Space Physics, vol. 118, pp. 73487359, doi: 10.1002/2013JA019099, 2013.

[5] S. Schaer, W. Gurtner and J. Feltens, "IONEX: The IONosphere Map Exchange Format Version 1," in Proceeding of the IGS AC Workshop, Darmstadt, Germany, 1998, pp 233-247. 
[6] M. Hernández-Pajares, J.M. Juan and J. Sanz, "Improving the Abel inversion by adding ground GPS data to LEO radio occultation in ionospheric sounding", Geophysical Research Letters, vol. 27, issue 16, pp. 2473-2476, 2000.

[7] B. Nanan, C.Y. Chen, P.K. Rajesh, J.Y. Liu and G.J. Bailey, "Modeling and observations of the low latitude ionosphere-plasmasphere system at long deep solar minimum", Journal of Geophysical Research. Space Physics, vol. 117, A08316, doi:10.1029/2012JA017846, 2012. 